\title{
On the unintentional rarefaction effect in LBM modeling of intrinsic permeability
}

\author{
Jun $\mathrm{Li}^{1 *}$, Minh Tuan $\mathrm{Ho}^{2}$, Lei $\mathrm{Wu}^{2}$, Yonghao Zhang ${ }^{2}$ \\ ${ }^{1}$ Center for Integrative Petroleum Research, College of Petroleum Engineering and Geosciences, \\ King Fahd University of Petroleum \& Minerals, Saudi Arabia \\ ${ }^{2}$ James Weir Fluids Laboratory, Department of Mechanical Engineering \& Aerospace Engineering,
}

University of Strathclyde, Glasgow, UK

(Received July 24, 2018; revised August 12, 2018; accepted August 13, 2018; available online August 22, 2018)

\section{Citation:}

Li, J., Ho, M.T., Wu, L., Zhang, Y. On the unintentional rarefaction effect in LBM modeling of intrinsic permeability. Advances in Geo-Energy Research, 2018, 2(4): 404-409, doi:

10.26804/ager.2018.04.05.

Corresponding author:

*E-mail: lijun04@gmail.com

Keywords:

Lattice Boltzmann method

pore-scale simulations

Knudsen number

\begin{abstract}
:
Lattice Boltzmann method (LBM) has been applied to predict flow properties of porous media including intrinsic permeability, where it is implicitly assumed that the LBM is equivalent to the incompressible (or near incompressible) Navier-Stokes equation. However, in LBM simulations, high-order moments, which are completely neglected in the Navier-Stokes equation, are still available through particle distribution functions. To ensure that the LBM simulation is correctly working at the Navier-Stokes hydrodynamic level, the high-order moments have to be negligible. This requires that the Knudsen number $(K n)$ is small so that rarefaction effect can be ignored. In our study, we elaborate this issue in LBM modeling of porous media flows, which is particularly important for gas flows in ultra-tight media. The influence of Reynolds number $(R e)$ on the intrinsic permeability is also discussed.
\end{abstract}

\section{Introduction}

Lattice Boltzmann method (LBM) is a popular method for calculation of flow properties of porous media e.g., intrinsic permeability (Ferréol and Rothman, 1995; Spaid and Phelan, 1997; Pan et al., 2006; Hosa et al., 2016; Prestininzi et al., 2016). The standard LBM is regarded as an alternative method to computational fluid dynamics (CFD), equivalent to solving the incompressible (or near incompressible) NavierStokes equation. Through the Chapman-Enskog expansion, we can show that the convergence of LBM to the incompressible Navier-Stokes equation in the low Mach and Knudsen numbers limits. However, these two methods are very different. For example, the third-order and higher-order moments are completely neglected in the isothermal Navier-Stokes equation while they are still available in LBM simulations through particle distribution functions, despite that they can be negligibly small when the Knudsen number $(K n)$ is close to zero. Therefore, the high-order moments have to be negligible if the LBM simulation is correctly working at the Navier-Stokes level, which has been commonly oversighted in simulating flows in porous media.

In LBM simulations, the model parameters can be correlated with the kinematic viscosity $v$ as follows:

$$
v=\frac{(\tau-0.5) c \Delta x}{3}
$$

where $\Delta x, \Delta t$ and $\tau$ are the grid or lattice length, the time step, and the normalized relaxation time, respectively; and $c=\Delta x / \Delta t$. Therefore, we have flexibility in selecting $c, \Delta x$, $\tau$ for any given physical $v$. In the following examples, we will demonstrate that with a given viscosity $v$, choice of time step, grid size and relaxation time may lead to finite $K n$, which incurs unintentional rarefaction effect at the NavierStokes level simulations. Therefore, appropriate combination of these model parameters can reduce the problem of viscositydependent permeability (Pan et al., 2006) even with singlerelaxation-time LBM. 


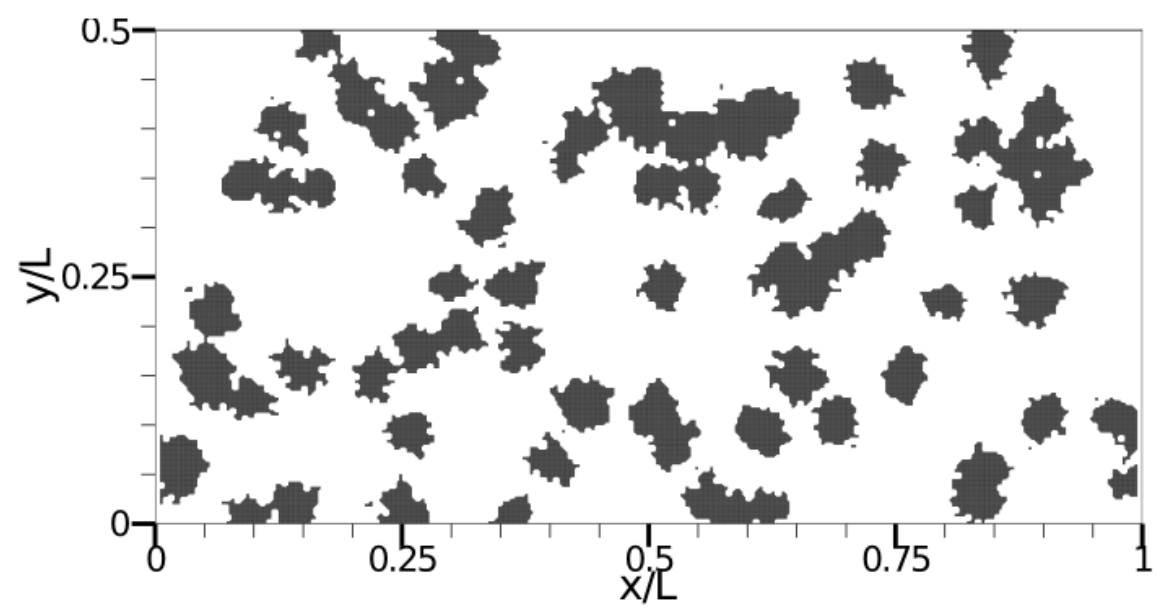

Fig. 1. The 2D geometry used in the pore-scale simulations, where the symmetric boundary condition is used at the top and bottom surfaces, while the pressure boundary condition is applied at the inlet and outlet.

\section{Single-relaxation-time Lattice Boltzmann and for D3Q19 model are}

\section{models}

The standard LBM with BGK collision operator (Qian et al., 1992) can be written as

$$
f_{\alpha}\left(\mathbf{x}+\Delta t \mathbf{e}_{\alpha}, t+\Delta t\right)=f_{\alpha}(\mathbf{x}, t)+\frac{f_{\alpha}^{\mathrm{eq}}(\mathbf{x}, t)-f_{\alpha}(\mathbf{x}, t)}{\tau}
$$

where the discrete equilibrium distribution function $f_{\alpha}^{\mathrm{eq}}$ is defined as

$$
f_{\alpha}^{\mathrm{eq}}=\omega_{\alpha} \rho\left[1+3 \frac{\mathbf{e} \cdot \mathbf{u}}{c^{2}}+\frac{9}{2} \frac{(\mathbf{e} \cdot \mathbf{u})^{2}}{c^{4}}-\frac{3}{2} \frac{u^{2}}{c^{2}}\right]
$$

Since we are interested in low-speed flows, the distribution function can be linearized around the equilibrium as $f=f^{\mathrm{eq}}(1+h)$. The governing Eq. (2) can be rewritten in terms of perturbed distribution function $h$ as follows

$$
h_{\alpha}\left(\mathbf{x}+\Delta t \mathbf{e}_{\alpha}, t+\Delta t\right)=h_{\alpha}(\mathbf{x}, t)+\frac{h_{\alpha}^{\mathrm{eq}}(\mathbf{x}, t)-h_{\alpha}(\mathbf{x}, t)}{\tau}
$$

where the perturbed equilibrium distribution function $h_{\alpha}^{\mathrm{eq}}$ is determined by

$$
h_{\alpha}^{\mathrm{eq}}=\omega_{\alpha}\left[\tilde{\rho}+3 \frac{\mathbf{e} \cdot \mathbf{u}}{c^{2}}\right]
$$

in which the high order terms of the flow velocity $\mathbf{u}$ are truncated. The discrete velocities for D2Q9 model are

$$
\mathbf{e}_{\alpha}=c \begin{cases}(0,0) & \alpha=0 \\ (1,0),(0,1),(-1,0),(0,-1) & \alpha=1-4 \\ (1,1),(-1,1),(-1,-1),(1,-1) & \alpha=5-8\end{cases}
$$

$$
\mathbf{e}_{\alpha}=c \begin{cases}(0,0,0) & \alpha=0 \\ ( \pm 1,0,0),(0, \pm 1,0),(0,0, \pm 1) & \alpha=1-6 \\ ( \pm 1, \pm 1,0),( \pm 1,0, \pm 1),(0, \pm 1, \pm 1) & \alpha=7-18\end{cases}
$$

The weights for $\mathrm{D} 2 \mathrm{Q} 9$ stencil are

$$
\omega_{\alpha}= \begin{cases}4 / 9 & \alpha=0 \\ 1 / 9 & \alpha=1-4 \\ 1 / 36 & \alpha=5-8\end{cases}
$$

and for D3Q19 are

$$
\omega_{\alpha}= \begin{cases}1 / 3 & \alpha=0 \\ 1 / 18 & \alpha=1-6 \\ 1 / 36 & \alpha=7-18\end{cases}
$$

The perturbed number density $\tilde{\rho}$ and the flow velocity $\mathbf{u}$ at a lattice point can be obtained by

$$
\tilde{\rho}=\sum_{\alpha} h_{\alpha}, \quad \mathbf{u}=\sum_{\alpha} \mathbf{e}_{\alpha} h_{\alpha}
$$

The rarefaction effect of the above LBM algorithm will be demonstrated in the following simulations of two-dimensional flows driven by pressure difference. Additionally, threedimensional flows driven by an external body force are also modeled below to study the rarefaction effect again and the force term is implemented by using the Shan-Chen model (Shan and Chen, 1993, 1994) as discussed in Section 2.2 of (Li and Brown, 2017).

\section{Results and discussions}

The intrinsic permeability depends on pore structure rather than the flow properties. First, we apply the D2Q9 LBM model to simulate a pressure-driven flow along the $x$ direction in a 


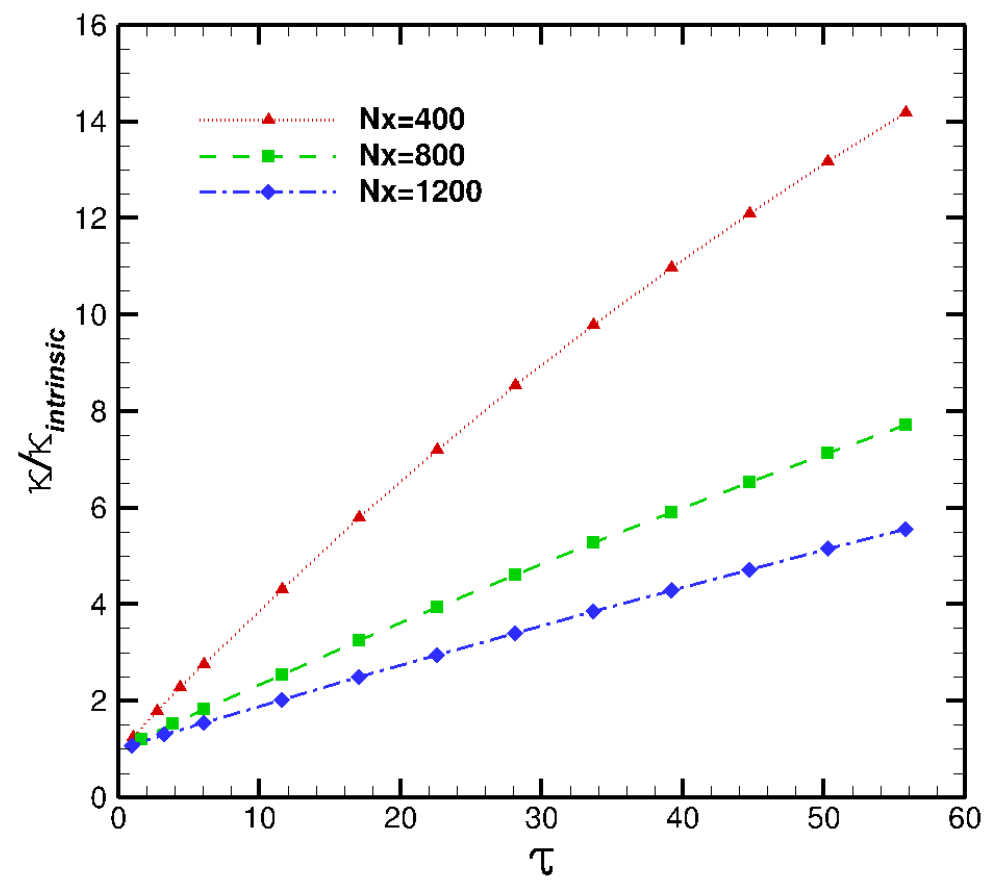

Fig. 2. The simulated permeability normalized by the intrinsic permeability against the relaxation time $\tau$ for 3 different resolutions.

2D randomly generated quartet structure (Wang et al., 2007), see Fig. 1. The applied pressure difference at the inlet and outlet is so small that the flows are in the Stokes flow regime. The permeability is then calculated according to the Darcy law with simulated mass flow rate. The porosity is 0.7394 and the resolutions are $N_{x} * N_{y}=400 * 200$, where $N_{x}$ and $N_{y}$ are the lattice numbers in the $x$ and $y$ directions. We also increase the resolutions to $800 * 400$, and $1200 * 600$ respectively. The porosity is also slightly changed to 0.7532 and 0.7578 respectively.

Fig. 2 shows that the permeabilities for 3 resolutions are very different even for the same $\tau$ and porous media. Only when $\tau-0.5$ is close to zero, they approach to their intrinsic permeability. Note, slightly different porosity will lead to very insignificant change to the intrinsic permeabilities. But the significantly different normalised permeabilities indicate that great care is required to choose right resolution and $\tau$. If we replot the normalized permeability against the Knudsen number (Zhang et al., 2005), which is

$$
K n=\sqrt{\frac{\pi}{6}} \frac{\tau-0.5}{N_{x}}
$$

for the D2Q9 and D3Q19 lattice models with single relaxation time (Qian et al., 1992), we find that the results also collapse into a single line especially for small $\mathrm{Kn}$, see Fig. 3. From Fig. 3 , we can clearly see the choice of parameters $\tau$ and $N_{x}$ should ensure that $K n<10^{-3}$ to simulate intrinsic permeability. Here, $K n$ is the global value based on the whole length of the domain i.e., $L$, and the local Knudsen number can be much larger to invalid Navier-Stokes level simulation even at $K n=0.01$. Although the choice of parameter range may be wider for multi-relaxation model, the underlying mechanism is still the same, i.e., LBM simulations of intrinsic permeability should choose appropriate $\tau$ and resolution i.e., $N_{x}$ to ensure $K n$ is small to exclude rarefaction effect.

In addition, Discrete Velocity Method (DVM) solution with very fine molecular velocity grid of 1,600 points (D2Q1600) serves as referenced solution in Fig. 3 to show the validity of LBM D2Q9 in the rarefied regime. Considering the same resolution of $N_{x} * N_{y}=800 * 400$, significant deviation of the LBM data (green curve) from the DVM data (black curve) can be seen when $K n \geq 5 \times 10^{-3}$ and grows up to $20 \%$ at $K n=5 \times 10^{-2}$. It is noted that both LBM and DVM use fully diffuse boundary condition on the solid wall to facilitate the comparison (Ansumali and Karlin, 2002; Wu et al., 2017).

We now use LBM D3Q19 to simulate a force-driven flow in a more realistic 3D porous media, see Fig. 4, as another example to study intrinsic permeability. The flows here are always driven in the $x$ direction, and the volumetric velocity $\langle\mathbf{u}\rangle_{\Omega}$ is used to compute the permeability $\kappa\left[\mathrm{m}^{2}\right]$ according to the Darcy law when an external force $\mathbf{g}=(g, 0,0)\left[\mathrm{m} / \mathrm{s}^{2}\right]$ is applied:

$$
\kappa=\frac{v\langle\mathbf{u}\rangle_{\Omega}}{g}=\frac{v}{g} \frac{\sum_{k \in \text { fluid }} \mathbf{u}_{k}}{N_{\text {grid }}}
$$

where $\sum_{k \in \text { fluid }} \mathbf{u}_{k}$ is summation of the flow velocity $\mathbf{u}_{k}$ over all fluid grids $k$, and $N_{\text {grid }}$ is the total grid number. We only show the permeability component in the driven direction in the following discussions the same as the above 2D case.

To calculate permeabilities of two similar geometries having the same $N_{\text {grid }}$ but $\Delta x_{2}=0.1 \Delta x_{1}$, we can select model parameters based on Eq. (1) to ensure both the Froude number (Fr) and Reynolds number $(R e)$ are the same, so that the calculated distribution functions will be the same. In these 


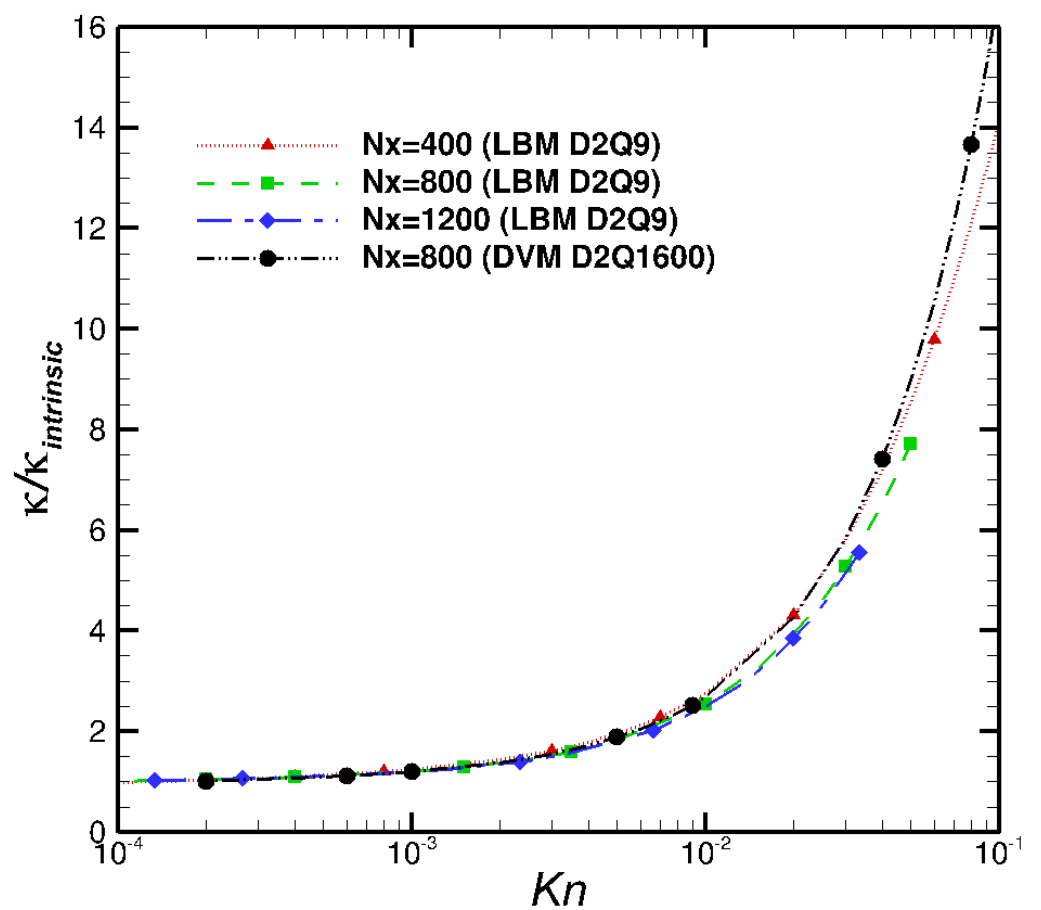

Fig. 3. The simulated permeability normalized by the intrinsic permeability against $K n$.

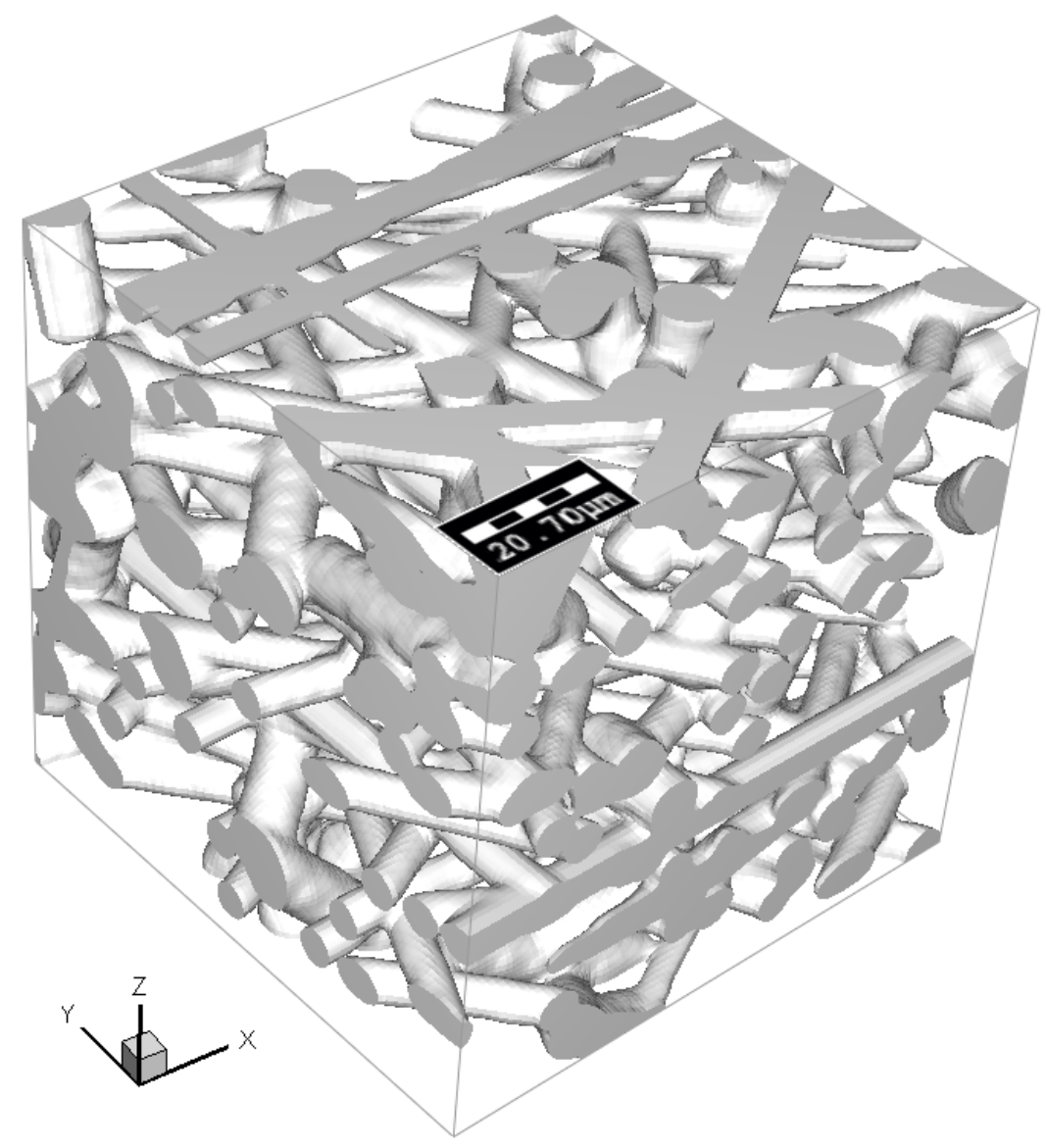

Fig. 4. The geometry used in the 3D pore-scale simulations. 


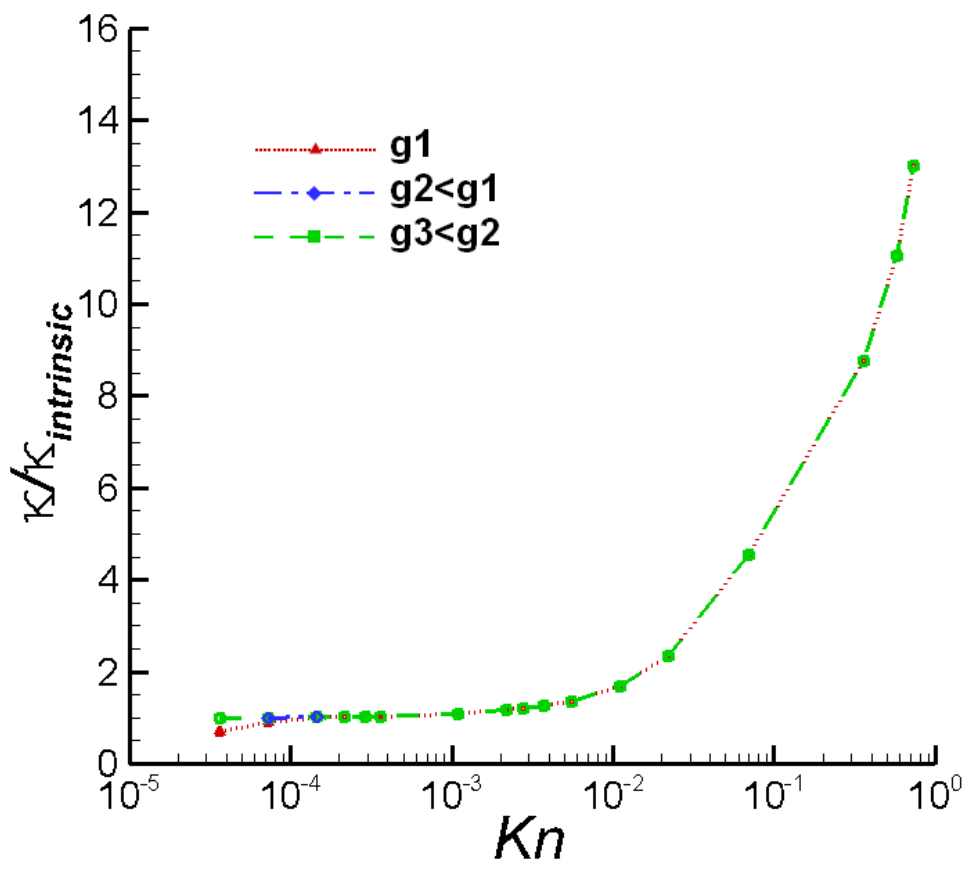

Fig. 5. Variation of permeability in the driven direction with $K n$ for different $g$.

two sets of parameters, one choice is to have $\Delta t_{2}=\Delta t_{1}$ (so $c_{2}=0.1 c_{1}$ ), $\tau_{2}=\tau_{1}$ (so $v_{2}=0.01 v_{1}$ according to Eq. (1)), and $g_{2}=0.1 g_{1}$. Thus, at the Navier-Stokes level, the velocity solutions of two cases satisfy $\mathbf{u}_{2}=0.1 \mathbf{u}_{1}$ and we have $\kappa_{2}=0.01 \kappa_{1}$ according to Eq. (12). Obviously, it is consistent with the following normalized incompressible Navier-Stokes equation:

$$
\frac{\partial \mathbf{u}^{\prime}}{\partial t^{\prime}}+\mathbf{u}^{\prime} \cdot \frac{\partial \mathbf{u}^{\prime}}{\partial \mathbf{x}^{\prime}}=-\frac{\partial\left(\frac{p}{\rho U^{2}}\right)}{\partial \mathbf{x}^{\prime}}+\frac{v}{U L} \Delta^{\prime} \mathbf{u}^{\prime}+\frac{L \mathbf{g}}{U^{2}}
$$

where $U$ and $L$ are the characteristic velocity and length to define the normalized parameters $t^{\prime}=t U / L, \mathbf{x}^{\prime}=\mathbf{x} / L$ and $\mathbf{u}^{\prime}=\mathbf{u} / U$. Eq. (13) also implies that more choices are possible to produce the same result (i.e., $\kappa_{2}=0.01 \kappa_{1}$ ), e.g., $\Delta t_{2}=0.1 \Delta t_{1} \quad\left(\right.$ so $\left.c_{2}=c_{1}\right), \tau_{2}=\tau_{1} \quad$ (so $v_{2}=0.1 v_{1}$ according to Eq. (1)), and $g_{2}=10 g_{1}$. Consequently, we have $\mathbf{u}_{2}=\mathbf{u}_{1}$ at the Navier-Stokes level. This analysis indicates that the same intrinsic permeability should be obtained with different choice of model parameters at the Navier-Stokes level.

However, both $\tau$ and $N_{\text {grid }}$ can affect calculated value of permeability as previously reported (Ferréol and Rothman, 1995; Pan et al., 2006; Hosa et al., 2016; Prestininzi et al., 2016) and demonstrated in the above 2D case. As Fig. 3 shows, it is actually through $K n$, which indicates how far the flow is away from the Navier-Stokes hydrodynamics. So we examine how $K n$ affect the calculation of intrinsic permeability in this 3D case.

The computational domain of Fig. 4 has $100^{3}$ voxels (i.e., grids) and the porosity $\phi$ of 0.748587 . The D3Q19 model is used with six periodic computational boundaries. Fig. 5 shows how the permeability changes with $K n$ for three different values of $g$. We can observe that the permeability is the same for different values of $g$ except at the small $K n$ for the large $g$, which is due to the increase of inertial effect while the kinematic viscosity decreases with $K n$ via $\tau$ at a fixed lattice velocity $c$, as previously reported in LBM simulations of pore-scale flows driven by pressure gradients (Sukop et al., 2013). The permeabilities of the cases with smaller $g$ will also drop if we keep decreasing $K n$ via $\tau$ leading to a significant increase of $R e$. Therefore, in addition to $K n$, inertial effect has to be checked in calculation of intrinsic permeability. If we remove the nonlinear velocity terms in equilibrium distribution function, equivalent to solving the Stokes equation i.e., the Navier-Stokes equation without the convective term ( $\mathrm{Li}$, and Brown, 2017), the permeability becomes the same for all three values of $g$.

\section{Conclusions}

Since the intrinsic permeability concerned here is defined by the Darcy law for small $R e$, where the viscosity effect is dominant, it is important to choose appropriate model parameters according to Eq. (1) to make sure both $R e$ and $K n$ are small in computing the intrinsic permeability. The resolution is usually low in pore-scale simulations to avoid high computational cost and thus the choice of $\tau$ becomes more restricted as we need to make sure both $R e$ and $K n$ are small. If we can afford high resolution, rarefaction effect may be excluded at a large $\tau$ by grid refinement.

\section{Acknowledgments}

The authors appreciate the valuable comments of the reviewers and editors.

Open Access This article is distributed under the terms and conditions of 
the Creative Commons Attribution (CC BY-NC-ND) license, which permits unrestricted use, distribution, and reproduction in any medium, provided the original work is properly cited.

\section{References}

Ansumali, S., Karlin, I.V. Kinetic boundary conditions in the lattice Boltzmann method. Phys. Rev. E 2002, 66(2): 026311.

Ferréol, B., Rothman, D.H. Lattice-Boltzmann simulations of flow through fontainebleau sandstone. Transp. Porous Media 1995, 20(1-2): 3-20.

Hosa, A., Curtis, A., Wood, R. Calibrating lattice Boltzmann ow simulations and estimating uncertainty in the permeability of complex porous media, Adv. Water Resour. 2016, 94: 60-74.

Li, J., Brown, D. Upscaled lattice Boltzmann method for simulations of flows in heterogeneous porous media. Geofluids 2017, 2017: 1740693.

Pan, C., Luo, L.S., Miller, C.T. An evaluation of lattice Boltzmann schemes for porous medium flow simulation. Comput. Fluids 2006, 35(8-9): 898-909.

Prestininzi, P., Montessori, A., Rocca, M.L., et al. Reassessing the single relaxation time lattice Boltzmann method for the simulation of Darcy's flows. Int. J. Mod. Phys. C 2016, 27(4): 1650037.
Qian, Y.H., d'Humieres, D., Lallemand, P. Lattice BGK models for Navier-Stokes equation. Europhys. Lett. 1992, 17(6): 479-484.

Shan, X., Chen, H. Lattice Boltzmann model for simulating flows with multi phases and components. Phys. Rev. E 1993, 47(3): 1815-1819.

Shan, X., Chen, H. Simulation of nonideal gases and liquidgas phase transitions by the lattice Boltzmann equation. Phys. Rev. E 1994, 49(4): 2941-2948.

Spaid, M.A., Phelan, F.R. Lattice Boltzmann methods for modeling microscale flow in fibrous porous media. Phys. Fluids 1997, 9(9): 2468-2474.

Sukop, M.C., Huang, H., Alvarez, P.F., et al. Evaluation of permeability and non-Darcy flow in vuggy macroporous limestone aquifer samples with lattice Boltzmann methods. Water Resour. Res. 2013, 49(1): 216-230.

Wang, M., Wang, J., Pan, N., et al. Mesoscopic predictions of the effective thermal conductivity for microscale random porous media. Phys. Rev. E 2007, 75(3): 1-10.

Wu, L., Ho, M.T., Germanou, L., et al. On the apparent permeability of porous media in rarefied gas flows. J. Fluid Mech. 2017, 822: 398-417.

Zhang, Y.H., Qin, R.S., Emerson, D.R. Lattice Boltzmann simulation of rarefied gas flows in microchannels. Phys. Rev. E 2005, 71(4): 047702. 